\title{
Zur (Un-)Möglichkeit von Teilhabe junger Menschen an der Gesellschaft
}

\section{Zwischen institutioneller Steuerung und Selbstorganisation von Interessenvertretung in Feldern der Kinder- und Jugendhilfe}

Die Kinder- und Jugendhilfe lässt sich als ein gesellschaftlich verantwortetes, dabei staatlich reguliertes Feld der institutionalisierten, professionellen Förderung, Unterstützung und Hilfe im Bereich der Sorge, Erziehung und Bildung für Familien, Kinder und Jugendliche bestimmen. Die rechtliche Grundlage bilden das Sozialgesetzbuch VIII (SGB VIII) und das Kinder- und Jugendstärkungsgesetz (KJSTG), die analog zur UN-Kinderrechtskonvention ein Recht auf Teilhabe junger Menschen beinhalten.

M it dem Begriff der Teilhabe ist wiederum ein sozialpolitischer Leitbegriff der letzten Jahrzehnte, aber auch ein Schlüsselbegriff einer gesellschaftlichen Selbstdeutung als Demokratie bezeich-

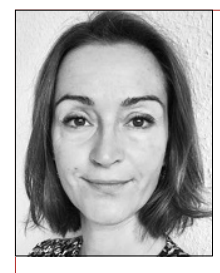

\section{Kathrin Maleyka}

Siegen, Deutschland

*1979. Staatsexamen Lehramt, wissenschaftliche Mitarbeiterin Fakultät II Department Erziehungswissenschaft, Universität Siegen.

Kathrin.Maleyka@uni-siegen.de

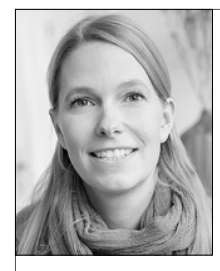

\section{Stefanie Bonus}

Technische Hochschule Köln, Köln, Deutschland Sozialarbeiterin (B.A.); M.A. Soziale Arbeit. Mitarbeiterin am Forschungsschwerpunkt Nonformale Bildung der TH Köln. Stefanie.bonus@th-koeln.de

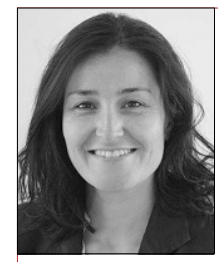

\section{Marcela Cano}

Technische Hochschule Köln, Köln, Deutschland Pädagogin der frühen Kindheit und Familienbildung (B.A.); M.A. Soziale Arbeit. Mitarbeiterin am Forschungsschwerpunkt Nonformale Bildung der TH Köln.

Marcela.cano@th-koeln.de

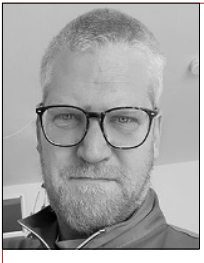

\section{Nils Wenzler}

Technische Hochschule Köln, Köln, Deutschland Dipl. Sozialpädagoge; M.A. Soziale Arbeit. Mitarbeitender am Forschungsschwerpunkt Nonformale Bildung der TH Köln. Nils.wenzler@th-koeln.de
Zusammenfassung Die Beiträge des Schwerpunkts „Interessenvertretung in der Kinder- und Jugendhilfe“ befassen sich mit den Möglichkeiten der Partizipation und Selbstorganisation, die Kindern und Jugendlichen in unterschiedlichen Feldern der Kinder- und Jugendhilfe eröffnet bzw. verwehrt sind. Obwohl solche Möglichkeiten durchaus eine rechtliche Verankerung haben, sind sie in der Praxis nur unzureichend umgesetzt. Wie die Beiträge verdeutlichen, liegen die Ursachen hierfür in Dilemmata, die nicht zuletzt in Strukturen der Kinder- und Jugendhilfe ihre Ursache haben. Aus fünf unterschiedlichen Perspektiven wird ein Einblick in jene strukturellen Dilemmata gegeben, die jeweils der Mitgestaltung junger Menschen im Hinblick auf ihre Lebensentwürfe und -umstände entgegenstehen; gleichzeitig werden Möglichkeiten deren produktiver Bearbeitung aufgezeigt.

Schlüsselwörter Strukturen der Kinder- und Jugendhilfe, Teilhabe, Selbstorganisation, Nutzer_innen

net. Sozialpolitisch beschreibt Teilhabe in den Beiträgen des Schwerpunkts das Verhältnis junger Menschen zu den sie betreffenden gesellschaftlichen Bedingungen ihres Aufwachsens. Der sozialpolitische Teilhabebegriff fragt sodann danach, „[...] inwiefern Menschen nach ihren eigenen Vorstellungen, die in der Gesellschaft vorfindbaren und gestaltbaren Optionen nutzen können (und nicht nutzen können) sowie tatsächlich nutzen (und nicht nutzen)“ (Bartelsheimer et al. 2020, S. 45).

Mit Blick auf die demokratische Gesellschaftsformation verweist Teilhabe auf die Zugänge und Öffnung von Berechtigungsräumen und das Recht auf die Mitgestaltung der gesellschaftlichen Bedingungen. Während der sozialpolitische Begriff es ermöglicht, die selbstbestimmte Lebensführung jeweils konkreter Individuen und deren partikularen Interessen in den Blick zu neh- 


\section{Extrablick: Interessenvertretung in der Kinder- und Jugendhilfe}

men, zielt die Perspektive der Berechtigungsräume auf gesellschaftliche Positionen von Individuen und Gruppen und deren Möglichkeit der Vertretung ihrer Interessen im Hinblick auf allgemeine Themen gesellschaftlicher Bedingungen des Aufwachsens. In diesem letzteren Fall geht es um die Ermöglichung aber auch Verwehrung von Teilhabe im Sinne einer Verteilungs- bzw. Umverteilungsfrage. „Es geht darum, dass in der Geschichte der modernen Demokratie die Freiheiten der einen regelmäßig die Zwänge der anderen waren, dass die Berechtigung der einen auf der Entrechtung anderer beruhte" (Lessenich 2019, S. 18).

Basierend auf einer demokratietheoretischen und demokratiegeschichtlichen Perspektive lässt sich fragen, inwieweit in der Kinder- und Jugendhilfe die Bereitschaft besteht, Berechtigungsräume zu öffnen, Selbstorganisation zu ermöglichen und eine (Selbst-)Vertretung der Interessen von Kindern und Jugendlichen zuzulassen. Teilhabe braucht, um wirksam zu sein, Verankerungen in den Institutionen, damit die Interessen von Kindern und Jugendlichen dort Gehör finden. Damit wird die Frage nach der (selbstorganisierten) Interessenvertretung von Kindern und Jugendlichen im Kontext des Systems der Kinder- und Jugendhilfe zu einer Frage nach den Möglichkeiten und Verwehrungen von Teilhabe im politischen Sinne. Grundlegend leitet sich der Anspruch auf Teilhabe für junge Menschen aus dem Grundgesetz $a b$, darüber hinaus formulieren die UN-Kinderrechtekonventionen den Anspruch auf Teilhabe aller Kinder und Jugendlichen an der Gestaltung ihrer Lebensverhältnisse (vgl. Bericht der Enquête-Kommission „Kinderschutz und Kinderrechte weiter stärken“ 2018). Anschlüsse an das Thema finden sich aber auch in den theorie-konzeptionellen Entwürfen Sozialer Arbeit, in der Rede vom ,Subjekt der Verhältnisse‘, Partizipation und Selbstbestimmung, oder in den fachpolitischen Debatten, unter den Schlagworten Mitbestimmung und Beteiligung (vgl. Deutscher Bundestag 2013, S. 77). Als aktuellen Anlass lassen sich vor allem zwei Punkte anführen, welche gleichermaßen auf der fachpolitischen Ebene angesiedelt sind:

- Erstens ist im 16. Kinder- und Jugendbericht der Bunderegierung neben dem Recht jedes jungen Menschen auf politische Bildung (S. 11) festgehalten, dass politische Teilhabe (bzw. Beteiligung) dazu beiträgt „[...] junge Menschen für die Demokratie zu gewinnen und sie zur demokratischen Teilhabe sowie zum Einsatz für gesellschaftlichen Zusammenhalt zu befähigen“ (Deutscher Bundestag 2020, S. 14). Darüber hinaus heißt es in der Stellungnahme der Bundesregierung, ,[...] dass Kinder und Jugendliche ihre sozialen Räume wirksam mitgestalten und über reale Konflik- te und Probleme mitentscheiden sollten und wird bestehende Regelungen daraufhin überprüfen, inwiefern die Mitbestimmung von jungen Menschen verbessert werden kann“ (Deutscher Bundestag 2020, S. 14).

- Zweitens lässt sich die Debatte zur Novellierung des SGB VIII anführen. Der Gesetzesentwurf der Bundesregierung (Bundesregierung 2017) sieht im Auftrag der Kinder- und Jugendhilfe in $\$ 1$ vor, „[...] die volle, wirksame und gleichberechtigte Teilhabe am Leben in der Gesellschaft für alle jungen Menschen zu verwirklichen [...]“ (Bundesregierung 2017, S. 10).

Auch hier wird als politischer Handlungsbedarf die Beteiligung an Entscheidungsprozessen aufgerufen: „Starke Kinder und Jugendliche brauchen mehr Beteiligung an den für ihr Aufwachsen maßgeblichen Entscheidungsprozessen: [erforderlich sind] bessere Beratungszugänge und erweiterte Beteiligungs- und Beschwerdemöglichkeiten für Kinder und Jugendliche [...]“ (Bundesregierung 2017, S. 1).

Die Bildung und Implementierung von Interessenvertretungen in der Kinder- und Jugendhilfe stellen zusammengefasst ein Desiderat dar. Ihre rechtliche Verankerung garantiert jedoch keineswegs eine unproblematische Umsetzung. In der Praxis zeigt sich vielmehr, dass wichtige Bedingungen einer Interessenvertretung junger Menschen in unterschiedlichen Feldern der Kinder- und Jugendhilfe bisweilen nur unzureichend gegeben sind. Wie die Beiträge des Themenschwerpunktes darlegen, liegt dieser „Lücke“ jeweils ein strukturelles Problem zugrunde:

Die ersten beiden Beiträge rücken Möglichkeitsräume der Teilhabe, die das Jugendverbandssystem jungen Menschen eröffnet, in den Blick. Stefanie Bonus, Marcela Cano und Nils Wenzler fokussieren in dem $\mathrm{Zu}$ sammenhang auf die Frage nach Teilhabemöglichkeiten, die das gegenwärtige Jugendverbandssystem jungen Menschen bietet, die sich in Selbstorganisationen zusammenfinden. Der Beitrag rückt die legitimatorischen Rahmungen des Jugendverbandssystems als staatlich geförderter Berechtigungsraum und die empirisch in Erscheinung tretenden Subjekte, die sich in Selbstorganisationen zusammenfinden, in ein Wirkungsgefüge. Was sich dabei zeigt, ist, dass systemimmanente Legitimationsstrukturen, weil ihnen ein regulierendes, kollektivierendes, zuweilen sanktionierendes Moment innewohnt, bisweilen einschränkend hinsichtlich der Möglichkeiten der Teilhabe junger Menschen in Form von Selbstorganisationen wirken.

Auch der zweite Beitrag von Deniz Greschner bewegt sich thematisch im Jugendverbandssystem. Hier wird der Fokus auf sogenannte „safer spaces“ in solchen 


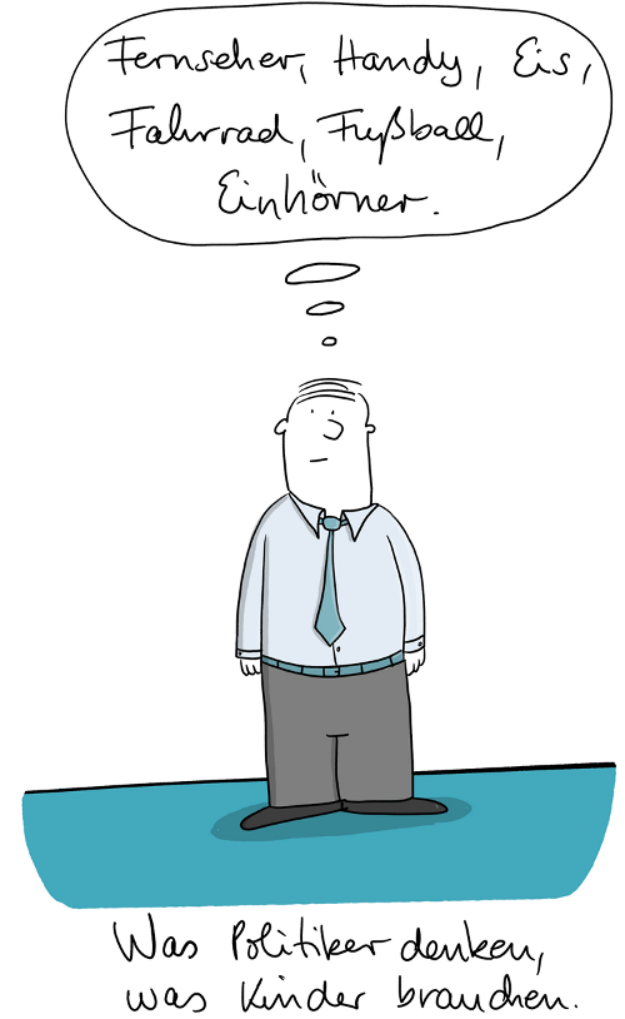

Jugendverbänden gelegt, die sich öffentlich als muslimisch positionieren. Safer spaces kommen, wie die Autorin ausgehend von ihrer eigenen Feldforschung zeigt, durchaus unterschiedliche Bedeutungen im Kontext muslimischer Jugendverbände zu: Sie stellen für junge Menschen Orte des politischen Bewusstseins und Engagements, aber auch Refugien dar, in denen Zuflucht vor defizitorientierte Zuschreibungen bieten, die aus Sicht der Autorin nicht zuletzt durch Strukturen der Jugendverbandsarbeit befeuert werden.

Die Frage nach Möglichkeiten der Partizipation und Selbstorganisation in der Offenen Kinder- und Jugendarbeit steht im Mittelpunkt des Beitrags von Benedikt Sturzenhecker und Moritz Schwerthelm. Die Autoren konstatieren die Förderung von Selbstorganisation benachteiligter Kinder und Jugendlichen als Aufgabe der Offenen Kinder- und Jugendarbeit, sehen diese Aufgabe jedoch zum Teil gerade dadurch nur unzureichend bearbeitet, dass pädagogische Fachkräfte Kindern und Jugendlichen Fähigkeiten der Selbstorganisation absprechen. Konsequente Förderung von Partizipation und Selbstorganisation setze aus Sicht von Sturzenhecker und Schwerthelm voraus, Kinder und Jugendliche nicht als hilfsbedürftig und insofern defizitär zu objektifizieren, sondern vielmehr ihre Fähigkeiten und Ressourcen anzuerkennen und damit Bedingungen für selbstbestimmtes und -organisiertes Handeln zu schaffen.
Tilman Lutz und Johannes Richter lenken in ihrem Beitrag den Fokus auf die Frage danach, inwiefern Ombudsstellen in der Kinder- und Jugendhilfe deren Adressat_innen erreichen. Ausgehend von eigenen empirischen Befunden zeigen die Autoren, dass Machtasymmetrien einerseits sowie Zugangshemmnisse andererseits es häufig verhindern, dass ombudschaftliche Beratungsangebote von jungen Menschen in Anspruch genommen werden. Ausgehend von dieser Befundlage plädieren Lutz und Richter für den Ausbau bzw. die Stärkung solcher Strukturen, die es den Adressat_innen von Kinder- und Jugendhilfe ermöglichen, ombudschaftliche Beratungsangebote als Ressource zu deuten und sie entsprechend aufzusuchen.

Ebenfalls thematisch im Feld der Kinder- und Jugendhilfe angesiedelt, befasst sich schließlich der Beitrag von Zoe Clark, Kathrin Maleyka und Arne Wohlfarth mit der Frage nach Voraussetzungen einer selbstorganisierten Interessenvertretung junger Menschen, die in stationären Hilfen zur Erziehung leben. Ausgehend von dem empirischen Befund, dass sich diese jungen Menschen zum Teil nicht als politisierte Subjekte und insofern als mitbestimmungsbefähigt konzeptualisieren. Die Autor_ innen schlussfolgern, dass es nicht zuletzt die Strukturen der Organisation sind, die die Herausbildung solcher Selbstkonzepte befördern, die wiederum der Partizipation an einer selbstorganisierten Interessenvertretung diametral entgegenstehen. 


\section{Extrablick: Interessenvertretung in der Kinder- und Jugendhilfe}

Die fünf Beiträge geben auf unterschiedliche Weise einen Einblick in strukturelle Dilemmata, die einer selbstbestimmten, selbstorganisierten Partizipation junger Menschen an der Mitgestaltung ihrer Lebensumstände entgegenstehen. Sie zeigen allerdings auch Möglichkeiten auf, diesen Dilemmata entgegenzuwirken und sind insofern als Impulse für die Praxis interpretierbar.

Eingegangen. 26. Juli 2021

Angenommen. 26. Juli 2021

Funding. Open Access funding enabled and organized by Projekt DEAL.

Open Access. Dieser Artikel wird unter der Creative Commons Namensnennung 4.0 International Lizenz veröffentlicht, welche die Nutzung, Vervielfältigung, Bearbeitung, Verbreitung und Wiedergabe in jeglichem Medium und Format erlaubt, sofern Sie den/die ursprünglichen Autor(en) und die Quelle ordnungsgemäß nennen, einen Link zur Creative Commons Lizenz beifügen und angeben, ob Änderungen vorgenommen wurden.

Die in diesem Artikel enthaltenen Bilder und sonstiges Drittmaterial unterliegen ebenfalls der genannten Creative Commons Lizenz, sofern sich aus der Abbildungslegende nichts anderes ergibt. Sofern das betreffende Material nicht unter der genannten Creative Commons Lizenz steht und die betreffende Handlung nicht nach gesetzlichen Vorschriften erlaubt ist, ist für die oben aufgeführten Weiterverwendungen des Materials die Einwilligung des jeweiligen Rechteinhabers einzuholen.

Weitere Details zur Lizenz entnehmen Sie bitte der Lizenzinformation auf http://creativecommons.org/licenses/by/4.0/deed.de.

\section{Literatur}

Bartelsheimer, P., et al. (2020). Teilhabe - eine Begriffsbestimmung. Wiesbaden: VS.

Bericht der Enquete-Kommission Kinderschutz und Kinderrechte (2018). Kinderschutz und Kinderrechte weiter stärken. Bürgerschaft der freien Hansestadt Hamburg (Hrsg.) Drucksache 21/16000 vom 19.12.2018

Bundesregierung (2017). Entwurf eines Gesetzes zur Stärkung von Kindern und Jugendlichen (Kinder- und Jugendstärkungsgesetz - KJSG). Gesetzentwurf der Bundesregierung. Bundestagsdrucksache 18/12330 vom 15.05.2017.

Deutscher Bundestag (2013). Bericht über die Lebenssituation junger Menschen und die Leistungen der Kinder- und Jugendhilfe in Deutschland

- 14. Kinder- und Jugendbericht - und Stellungnahme der Bundesregierung. Bundestagsdrucksache 17/12200 vom 30.01.2013.

Deutscher Bundestag (2020). Bericht über die Lage junger Menschen und die Bestrebungen und Leistungen der Kinder- und Jugendhilfe - 16. Kinderund Jugendbericht - Förderung demokratischer Bildung im Kindes- und Jugendalter und Stellungnabme der Bundesregierung. Bundesdrucksache 19/24200 vom 11.11.2020.

Lessenich, S. (2019). Grenzen der Demokratie. Teilhabe als Verteilungsproblem. Stuttgart: Reclam.

Hier steht eine Anzeige.

Springer 